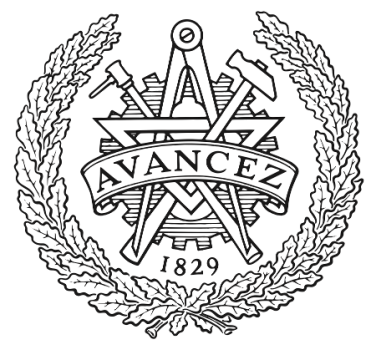

CHALMERS

UNIVERSITY OF TECHNOLOGY

\title{
Local Coordination Environments and Vibrational Dynamics of Protons in Hexagonal and Cubic Sc-Doped BaTiO3 Proton-Conducting Oxides
}

Downloaded from: https://research.chalmers.se, 2023-04-26 09:08 UTC

Citation for the original published paper (version of record):

Perrichon, A., Torino, N., Jedvik Granhed, E. et al (2020). Local Coordination Environments and Vibrational Dynamics of Protons in Hexagonal and Cubic

Sc-Doped BaTiO3 Proton-Conducting Oxides. Journal of Physical Chemistry C, 124(16): 8643-8651. http://dx.doi.org/10.1021/acs.jpcc.0c01705

N.B. When citing this work, cite the original published paper. 


\section{Local Coordination Environments and Vibrational Dynamics of Protons in Hexagonal and Cubic Sc-Doped $\mathrm{BaTiO}_{3}$ Proton- Conducting Oxides}

Adrien Perrichon,* Nico Torino, Erik Jedvik Granhed, Yuan-Chih Lin, Stewart F. Parker, Mónica Jiménez-Ruiz, Maths Karlsson, and Paul F. Henry

Cite This: J. Phys. Chem. C 2020, 124, 8643-8651

Read Online

ACCESS | Lلll Metrics \& More | 回 Article Recommendations | st Supporting Information

ABSTRACT: The proton local coordination environments and vibrational dynamics associated with the two order of magnitude change in proton conductivity in hydrated forms of hexagonal and cubic structured $\mathrm{BaTi}_{1-x} \mathrm{Sc}_{x} \mathrm{O}_{3} \mathrm{H}_{x}(0.16<x<0.7)$ were investigated using optical spectroscopy, neutron spectroscopy, and firstprinciples calculations. Whereas the cubic structure compositions display a single proton site, we show that protons occupy three distinct sites in compositions exhibiting the hexagonal structure. The principal site is characterized by interoctahedral hydrogen

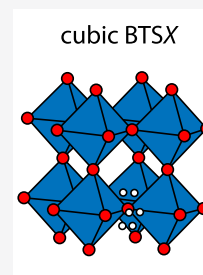

one proton site
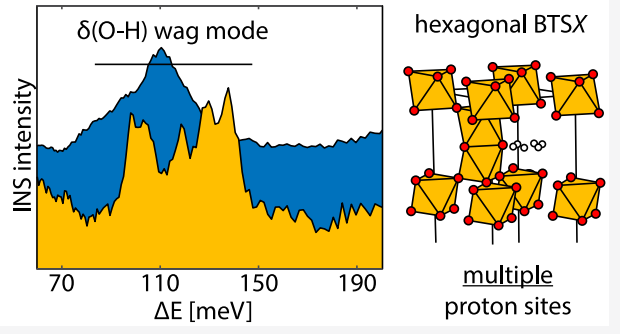
bonds, while two additional low occupancy sites are similar to those in the cubic structure, with classic intraoctahedral geometry. Furthermore, the proton hydrogen bond strength increases with decreasing scandium doping level. We infer from this that the stronger, more energetic hydrogen bonds in the hexagonal structure, resulting from proton sites with lower symmetry (lower multiplicity), are predominantly responsible for the significant reduction in macroscopic conductivity between cubic and hexagonal $\mathrm{BaTi}_{1-x} \mathrm{Sc}_{x} \mathrm{O}_{3} \mathrm{H}_{x}$ materials, rather than simply the absolute number of protons. Our findings are highly relevant to the field, clarifying the advantages of high-symmetry structures with high-multiplicity proton sites to favorable properties in ceramic proton-conducting oxides.

\section{INTRODUCTION}

Proton-conducting oxides find uses in many energy-related applications, including membranes for steam methane reforming, ${ }^{1,2}$ steam electrolysis, ${ }^{3,4}$ and hydrogen separation, ${ }^{5}$ or as electrolyte materials in a solid-state proton-conducting fuel cell (PCFC). ${ }^{6-15}$ The development of the PCFC technology depends on the discovery of novel materials with high proton conduction in the intermediate temperature regime, $T=200-600{ }^{\circ} \mathrm{C}$. Acceptor-doped perovskites, based on barium zirconate, $\mathrm{BaZrO}_{3}$, and barium cerate, $\mathrm{BaCeO}_{3}$, are currently the most studied electrolyte candidates, due to good thermal and chemical stability in addition to high proton conductivity. ${ }^{11-17}$ Materials based on titanates, such as acceptor-doped $\mathrm{BaTiO}_{3}{ }^{21-26}$ and Ti-doped $\mathrm{Ba}_{2} \mathrm{In}_{2} \mathrm{O}_{5}{ }^{18-20}$ are less well-studied, yet show great promise. Of particular interest to this work is the solid solution of scandium doping into barium titanate, ${ }^{25} \mathrm{BaTi}_{1-x} \mathrm{Sc}_{x} \mathrm{O}_{3-x}$ (BTS $X$ where $X$ is the scandium doping $x$ expressed as a percentage from $0-100$ ), assuming ideal substitution of $\mathrm{Sc}^{3+}$ for $\mathrm{Ti}^{4+}$.

The BTSX solid solution $(0<X<70)$ exhibits a structural phase transition from a $6 \mathrm{H}$ hexagonal perovskite to a cubic perovskite phase with increasing $X$. In hexagonal materials, cations distribute over two sites: corner-sharing $\mathrm{MO}_{6}$ octahedra and face-sharing $\mathrm{M}_{2} \mathrm{O}_{9}$ polyhedra where $\mathrm{M}=\mathrm{Sc}$ and Ti. Only corner-sharing $\mathrm{MO}_{6}$ octahedra occur in the cubic variants. $^{25}$ In all cases, the oxygen-deficient materials fully hydrate under humid conditions, yielding $\mathrm{BaTi}_{1-x} \mathrm{Sc}_{x} \mathrm{O}_{3} \mathrm{H}_{x}$, which are good proton conductors, reaching a maximum conductivity of $2 \times 10^{-3} \mathrm{~S} \mathrm{~cm}^{-1}$ at $600{ }^{\circ} \mathrm{C}$ for the $x=0.7$ composition. ${ }^{24}$ The proton conductivity in the hexagonal materials is at least 2 orders of magnitude lower with respect to cubic materials. There is also a high activation energy for bulk proton conduction of about $0.77-0.80 \mathrm{eV}^{25,26}$

Recent further structural work, using neutron powder diffraction (NPD) on BTS20 and BTS70 compositions, ${ }^{27}$ shows cation ordering in the hexagonal structure composition, with scandium only occupying the corner-sharing $\mathrm{MO}_{6}$ octahedra, implying an upper limit at $X=33(x=0.33)$ before transition to the cubic structure. No long-range metal order occurs in the cubic structure. In the dehydrated, host materials, oxygen vacancies localize in the shared face of the $\mathrm{M}_{2} \mathrm{O}_{9}$ polyhedra in the hexagonal structure and show no long-

Received: February 27, 2020

Revised: April 1, 2020

Published: April 14, 2020 

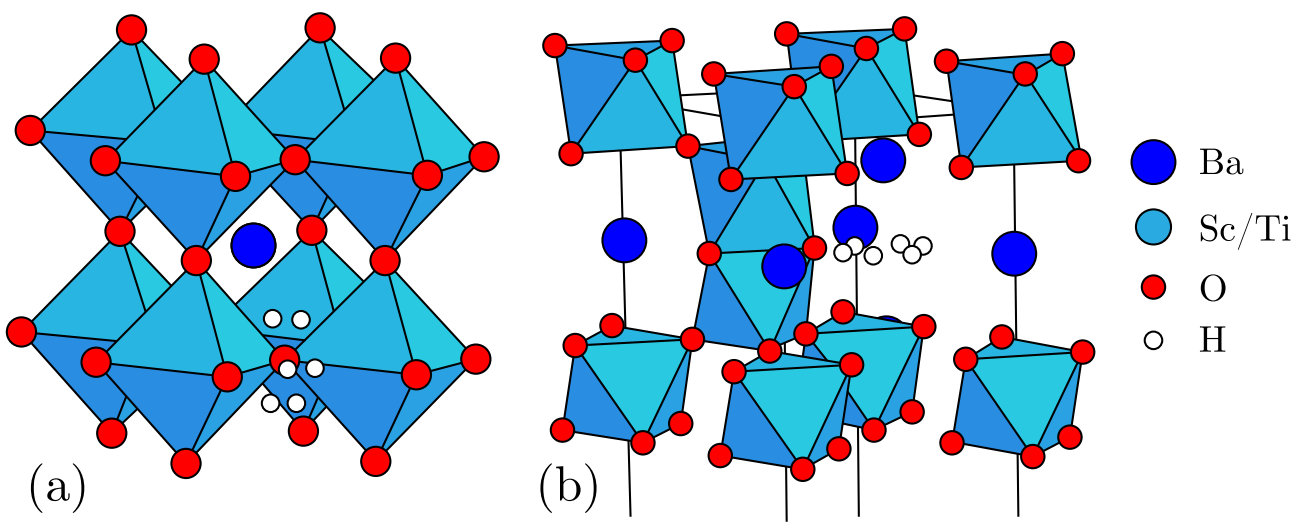

Figure 1. Scheme of the cubic structure (a) and top half of the hexagonal structure (b) of hydrated BTSX.

range order in the cubic structure. Figure 1 shows the hydrated hexagonal and cubic structures from room temperature NPD by Torino et al., ${ }^{27}$ which demonstrate significantly different proton sites for the two structural types. Additionally, in situ dehydration of BTS50 and BTS70, ${ }^{28}$ which are both cubic, shows significantly different dehydration rates and response of the lattice parameter as a function of temperature during the dehydration process, indicative of differing hydrogen bond strengths in the two materials. The structural investigations suggest that the proton site geometry in the hexagonal phase negatively influences proton mobility, in addition to the reduced number of protons present per formula unit. It also suggests a secondary contribution to the proton mobility related to the total number of protons and their interaction with the oxygen sublattice.

Here, we present a detailed characterization of proton vibrational dynamics, to investigate the different contributions, in four compositions of hydrated $\mathrm{BaTi}_{1-x} \mathrm{Sc}_{x} \mathrm{O}_{3} \mathrm{H}_{x}$ across both structure types, with $x=0.16,0.20$ (hexagonal) and $x=0.50$ and 0.70 (cubic), hereafter referred to as BTS16, BTS20, BTS50, and BTS70 respectively. We report results from Raman spectroscopy, inelastic neutron scattering (INS), and infrared (IR) spectroscopy, which give access to different regions of the vibrational spectra: the lattice dynamics, the hydroxide wag bands, $\delta(\mathrm{O}-\mathrm{H})$, and the hydroxide stretch bands, $\nu(\mathrm{O}-\mathrm{H})$. We also report results from first-principles density functional theory (DFT) calculations, based on structures determined by NPD. ${ }^{27}$ The purpose of the study is to evaluate the impact of the scandium content and crystal structure, i.e., the influence of the phase transition, on the number and relative occupation of distinct proton sites in $\mathrm{BaTi}_{1-x} \mathrm{Sc}_{x} \mathrm{O}_{3} \mathrm{H}_{x}$ to obtain insights into the relationship between these properties and the proton conductivity.

\section{METHODS}

Sample Preparation. All samples were prepared using solid-state reaction methods described in detail in references 27 and 28. The starting materials were $\mathrm{BaCO}_{3}$ (Alfa Aesar, 99.8\%), $\mathrm{TiO}_{2}$ (Sigma-Aldrich, 99.8\%), and $\mathrm{Sc}_{2} \mathrm{O}_{3}$ (Alfa Aesar, 99.9\%). Final sintering temperatures for the four samples were $1425{ }^{\circ} \mathrm{C}$ for BTS16, $1410{ }^{\circ} \mathrm{C}$ for BTS20, and $1500{ }^{\circ} \mathrm{C}$ for BTS50 and BTS70. After the sintering, the samples were vacuum-dried in a furnace at $500{ }^{\circ} \mathrm{C}$ for $96 \mathrm{~h}$, inside a quartz tube, under dynamic vacuum at $1 \times 10^{-6}$ mbar. Sample hydration in a flow-tube furnace assembly used nitrogen (Air Liquide Alphagaz 1, containing 6 ppm of $\mathrm{H}_{2} \mathrm{O}$ ) as the carrier gas, after bubbling through distilled water. See references 27 and 28 for details of the hydration process and precharacterization of the samples by diffraction and thermogravimetric techniques.

Infrared Spectroscopy. IR spectroscopy measurements were performed in diffuse reflectance mode using a Thermo Scientific Nicolet 6700 FT-IR spectrometer equipped with a Collector II accessory for diffuse reflection measurements. IR spectra were collected on the four samples (ca. $50 \mathrm{mg}$ each) at room temperature, over the energy range $1200-4000 \mathrm{~cm}^{-1}$. The energy resolution over that interval was set to $2 \mathrm{~cm}^{-1}$. The spectrum of a rough $\mathrm{Au}$ mirror was used as the reference spectrum. Absorbance-like spectra were derived by taking the logarithm of the ratio between the reference and sample spectra. All measurements were performed at room temperature.

Raman Spectroscopy. Raman spectra were measured on the four samples at $300 \mathrm{~K}$, using a Renishaw InVia Reflex spectrometer. A $532 \mathrm{~nm}$ laser was used as the excitation source and the laser beam was focused on the sample through a $\times 50$ objective lens $(\mathrm{NA}=0.5)$. The power of the laser beam impinging on the sample was approximately $1 \mathrm{~mW}$. The light backscattered from the sample was then collected through the same objective lens and was dispersed by a $24001 / \mathrm{mm}$ grating prior to the detection by a CCD camera.

Inelastic Neutron Scattering. The INS experiments were performed on the IN1 Lagrange spectrometer at the Institut Laue-Langevin, France, and on the TOSCA and MAPS spectrometers at the ISIS Facility, U.K.

The indirect-geometry spectrometer IN1 Lagrange was used to measure about $5 \mathrm{~g}$ of the BTS20 and BTS70 samples, respectively, placed in cylindrical hollow aluminum cells. Using a combination of monochromator crystals, the spectra were collected over the energy range $\hbar \omega=0-500 \mathrm{meV}$, and with increased statistics with the $\mathrm{Cu}(220)$ monochromator over the extended $\delta(\mathrm{O}-\mathrm{H})$ mode region, $\hbar \omega=50-200 \mathrm{meV}$. The indirect-geometry spectrometer TOSCA was used to complement the data set by measuring over the extended $\delta(\mathrm{O}-\mathrm{H})$ mode region the BTS16 (5.9 g), BTS20 (5.0 g), BTS50 (2.1 g) and BTS70 (5.2 g) samples, which were placed in flat aluminum cells and sealed with indium wires. The directgeometry spectrometer MAPS was used to access the extended $\nu(\mathrm{O}-\mathrm{H})$ mode region, $\hbar \omega=300-600 \mathrm{meV}$, for the four samples and with the same sample cells used on TOSCA. Spectra were collected with incident neutron energies of $E_{i}=$ $650 \mathrm{meV}(600 \mathrm{~Hz})$ and $E_{i}=300 \mathrm{meV}(400 \mathrm{~Hz})$, respectively, 
where the number in parentheses refers to the Fermi chopper frequency.

All the measurements were performed at $T<20 \mathrm{~K}$. Reduction and standard corrections of the data were performed using the LAMP ${ }^{29}$ software for the IN1 Lagrange data, and the MANTID ${ }^{30}$ software for the TOSCA and MAPS data.

Computational Details. DFT calculations were performed using the projector augmented wave (PAW) method $^{31,32}$ as implemented in the VASP ${ }^{33,34}$ software together with the $\mathrm{PBE}^{35}$ exchange-correlation functional. For the cubic $\mathrm{BaTiO}_{3}$ a supercell containing 40 atoms constructed from a 2 $\times 2 \times 2$ stacking of primitive unit cells was used in most calculations. We denote this the defect cell. The introduction of a proton in the defect cell implies a defect concentration of $12.5 \%$. The same defect cell was used for the calculation of the combined defect of a proton and a scandium atom in various configurations. For hexagonal $\mathrm{BaTiO}_{3}$, the unit cell containing 30 atoms was used as the defect cell implying a defect concentration of $16.7 \%$. For the defect cell a Monkhorst-Pack $k$-point mesh of $6 \times 6 \times 6$ for the cubic and a $\Gamma$-centered $6 \times 6$ $\times 2$ for the hexagonal cell were used together with a plane wave cutoff energy of $700 \mathrm{eV}$. The calculations were converged to energies within $10^{-7} \mathrm{eV}$ for the electronic structure and to forces within $10^{-3} \mathrm{eV} / \AA$ for the ionic relaxations. The eigenmodes were obtained using the frozen phonon method in the harmonic approximation as implemented in phonopy. ${ }^{36}$ Calculations of the force constants of the dynamical matrix were conducted in the defect cell.

\section{RESULTS AND DISCUSSION}

Infrared Spectroscopy. The IR spectra of the four samples are shown in Figure 2. The main feature of the spectra is the band in the region $350-450 \mathrm{meV}$, characteristic of the $\nu(\mathrm{O}-\mathrm{H})$ modes in hydrated oxides and particularly in

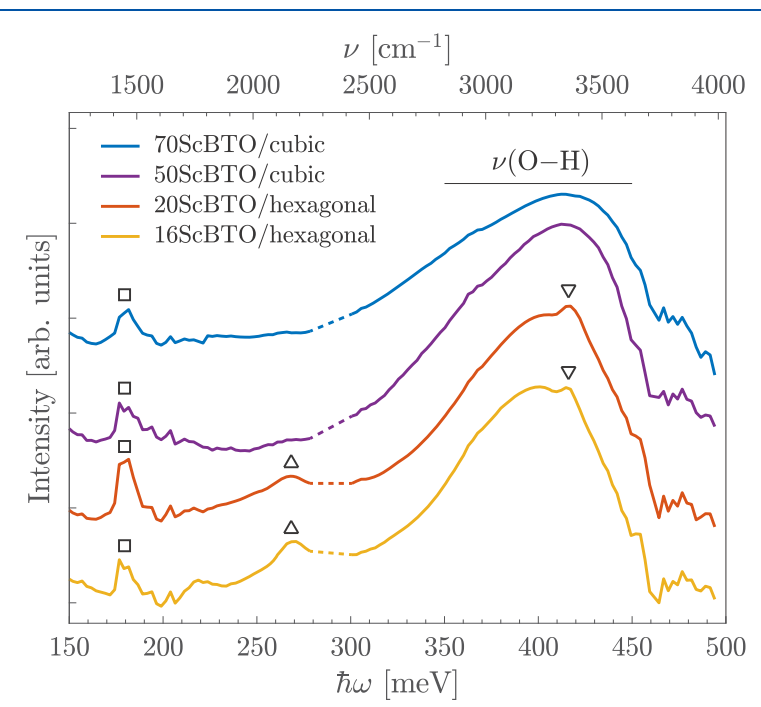

Figure 2. IR spectra of BTS70 (blue), BTS50 (purple), BTS20 (orange), and BTS16 (yellow). The $276-300 \mathrm{meV}$ region of the spectra are omitted due to a strong absorption of the Au reference; interpolated dashed lines are instead shown as guide for the eyes. The peak at $c a .180 \mathrm{meV}$ (squares) is assigned to a carbonated impurity. ${ }^{43}$ The spectra have been corrected by subtraction of a linear background and normalization to the integral of the $\nu(\mathrm{O}-\mathrm{H})$ band $(350-450 \mathrm{meV})$ and are vertically offset for clarity. hydrated acceptor-doped cubic perovskite oxides. ${ }^{37-40}$ The overall downward shift of the band in comparison with the free $\mathrm{O}-\mathrm{H}$ molecular ion $\nu(\mathrm{O}-\mathrm{H})$ energy, at about $450 \mathrm{meV},{ }^{41}$ is attributed to the presence of hydrogen bonds. ${ }^{42}$ The large width of the band is then rationalized by a distribution in strength of the hydrogen bonds, underpinning the coexistence of numerous proton local environments. The asymmetry of the band toward lower energy may indicate the presence of a distinct second population of proton sites, or it may be an effect of the increased IR cross-section of the increasingly polarized $\mathrm{O}-\mathrm{H}$ unit when its hydrogen bond is strengthened. The main $\nu(\mathrm{O}-\mathrm{H})$ band is centered at about $420 \mathrm{meV}$ for BTS50 and BTS70, $410 \mathrm{meV}$ for BTS20, and $405 \mathrm{meV}$ for BTS16, which denotes an overall strengthening of the hydrogen bonds in the hexagonal phases.

The spectra of the BTS 16 and BTS20 samples show a more defined $\nu(\mathrm{O}-\mathrm{H})$ band than the cubic counterparts. They also contain minor peaks at about 270 and $418 \mathrm{meV}$ (triangle up and down in Figure 2). This indicates that, while the spread of hydrogen bond environments may be lower in the hexagonal phases with respect to the cubic phases, additional distinct proton sites are present.

Raman Spectroscopy. The Raman spectra of the four samples are shown in Figure 3. The presence of bands in the

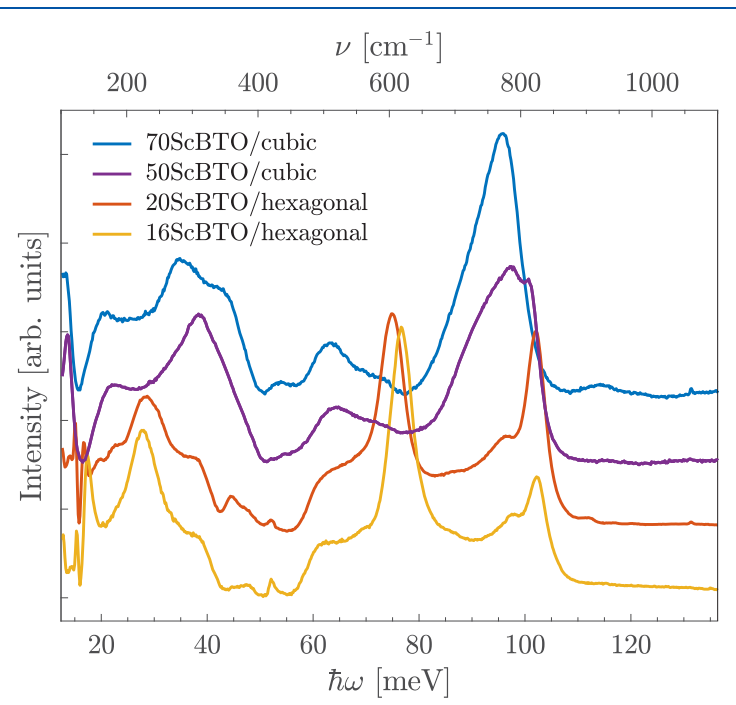

Figure 3. Raman spectra of BTS70 (blue), BTS50 (purple), BTS20 (orange), and BTS16 (yellow), over the lattice mode region. The spectra have been corrected by the subtraction of a linear background, have been normalized to unity, and are vertically offset for clarity.

Raman spectra of cubic BTS50 and BTS70, which should be forbidden based on the group theory analysis of the crystal structure with perfect cubic symmetry ( $P m \overline{3} m$ space group), is rationalized by symmetry breaking at the local scale induced by the occupational disorder between $\mathrm{Sc}$ and $\mathrm{Ti}$ over the $B$-site of the $A B \mathrm{O}_{3}$ perovskite structure, and by the presence of oxygen vacancies. This local disorder effect on Raman modes is also commonly observed for the cubic phase of undoped $\mathrm{BaTiO}_{3}$ (BTO) ( $P m \overline{3} m$ space group), which is usually transformed from the tetragonal phase $(P 4 \mathrm{~mm})$ at ambient pressure and room temperature after applying high pressures $(\sim 2-3.5 \mathrm{GPa})$ or temperatures $(\sim 395-405 \mathrm{~K}))^{44-46}$ The energies of the modes are extracted by peak fit analysis of the Raman spectra with Gaussian functions, and reported in Table 1. 
Table 1. Mode Energies, in meV, Extracted by Peak Fit Analysis of the Raman Spectra ${ }^{a}$

$\begin{array}{ccccc} & \text { BTS70 } & \text { BTS50 } & \text { BTS20 } & \text { BTS16 } \\ 1 & 19.8(1)^{\prime} & 21.5(4)^{\prime} & 21.3(2) & - \\ 2 & 24.7(3) & 28.3(5) & 28.7(2)^{\prime} & 27.4(3)^{\prime} \\ 3 & 35.4(1)^{\prime} & 38.7(3)^{\prime} & 37.8(2) & 37.3(5) \\ 4 & 43.8(1) & 45.8(4) & 44.5(5) & 45.0(2) \\ 5 & - & - & 47(1) & 47.4(2) \\ 6 & 53.7(2) & 54.4(1) & 52.0(6) & 52.6(1) \\ 7 & 63.9(2)^{\prime} & 63.3(1)^{\prime} & 63.2(1) & 64.3(1) \\ 8 & 72.7(2) & 72.1(3) & 74.2(1)^{\prime} & 76.5(1)^{\prime} \\ 9 & 85.3(4) & 88.7(4) & 86.5(3) & 86.9(2) \\ 10 & 94.5(2)^{\prime} & 96.2(3)^{\prime} & 94.9(3) & 96.9(7) \\ 11 & - & 100.8(4) & 102.0(2)^{\prime} & 102.7(2)^{\prime} \\ 12 & 114.0(3) & - & 111.1(2) & -\end{array}$

${ }^{a}$ Standard error are indicated in parentheses. Main peaks are indicated with a single prime.

Overall, we observe minor differences between the Raman spectra of the two cubic samples, BTS50 and BTS70. The double peak at $35-45 \mathrm{meV}$ in BTS70 appears as a single peak at $40 \mathrm{meV}$ in BTS50. The highest-energy band at $\sim 95 \mathrm{meV}$ appears as a narrower band with a single peak in BTS70, but as a wider doublet band in BTS50. These differences in the band shapes and positions may arise from the changes in the local $\mathrm{B}-\mathrm{O}$ bond lengths and/or angles of $\mathrm{BO}_{6}$ octahedral units as a result of increasing the concentration of the larger $\mathrm{Sc}^{3+}$ dopant, whose ionic radius is about $23 \%$ larger than the $\mathrm{Ti}^{4+}$ one. Note that the spectral differences may be less likely related to the mass effect since the masses of $\mathrm{Sc}^{3+}(45 \mathrm{u})$ and $\mathrm{Ti}^{4+}(48 \mathrm{u})$ are comparable. Nonetheless, the Raman spectra of BTS50 and BTS70 are in general similar, which indicates that the local structures and associated lattice dynamics of these materials are almost the same. Interestingly, the Raman spectra of cubic BTS50/BTS70 and cubic BTO exhibit some similarities, as characterized by two main groups of bands in the spectral ranges of $<50$ and $\sim 50-75 \mathrm{meV}$, respectively, except for the absence of bands at $>75 \mathrm{meV}$ in cubic BTO. ${ }^{44-46}$

The Raman spectra of the two hexagonal samples, BTS16 and BTS20, are also similar to each other, except for the $\sim 2$ $\mathrm{meV}$ downward shift of the two bands at $75 \mathrm{meV}$ and at 96
$\mathrm{meV}$ on increasing the Sc concentration. Specifically, the band at $74.2 \mathrm{meV}$ for BTS20 (76.5 meV for BTS16) is one of the key Raman mode features for hexagonal perovskite oxides. $^{47-49}$ Moreover, the Raman spectra of BTS16 and BTS20 in the spectral range of $<90 \mathrm{meV}$ are very similar to the Raman spectrum of hexagonal BTO, which is mainly characterized by three strong bands at 19.5, 27.9, and 79.7 $\mathrm{meV}^{49}$

In summary, both cubic and hexagonal phases of Sc-doped BTO show an additional band feature in the high energy regions ( $>75$ and $>90 \mathrm{meV}$ for the cubic and hexagonal phases, respectively), in comparison with the corresponding phases of undoped BTO, which suggests that the local disorder specifically due to the Sc doping effect induces Raman-active modes. Regardless of different crystal structures, this high energy band exhibits a general energy downward shift upon increasing the Sc concentration from BTS16 to BTS70. This implies that these high-energy modes in the four samples studied may originate from the same type of local disorder that is virtually independent of the structural phases but it is primarily influenced by the amount of Sc dopant.

Indeed, qualitatively similar spectra are recorded in the system $\mathrm{BaZr}_{x} \mathrm{Ti}_{1-x} \mathrm{O}_{3}$ with $0.5 \leq x \leq 1.0$, where the $B$-site is shared by $\mathrm{Zr}^{4+}$, similar in size to $\mathrm{Sc}^{3+}$ (72 and $74 \mathrm{pm}$, respectively, when hexacoordinated). ${ }^{50,51}$ An intense band at $\sim 90 \mathrm{meV}$ is observed and discussed in the context of the cubic to tetragonal phase transition, where the symmetric $\mathrm{Ti}-\mathrm{O}-\mathrm{Ti}$ unit in the cubic phase becomes asymmetric in the tetragonal phase, $\mathrm{Ti}-\mathrm{O} \cdot \mathrm{Ti}$, with longer and shorter $\mathrm{Ti}-\mathrm{O}$ bonds. This $\sim 90 \mathrm{meV}$ band is assigned to an $\mathrm{A}_{1}$ ( $\mathrm{LO}$ ) mode, the stretch of the shorter $\mathrm{Ti}-\mathrm{O}$ bonds. It is also observed in orthorhombic and rhombohedral $\mathrm{BaTiO}_{3}$, but with a weaker intensity. ${ }^{52}$ In BTS50 and BTS70, the occupational disorder between Sc and Ti over the $B$-site leads necessarily to a number of asymmetric $B-\mathrm{O} \cdot B$ units and, thus, a distribution of $B-\mathrm{O}$ bond lengths. This effect is also present for BTS16 and BTS20 to a lesser degree, as only the $(2 a) B$-sites are concerned by the occupational disorder. By analogy, we thus tentatively assign the $\sim 95 \mathrm{meV}$ band, present in the four samples, to $\mathrm{A}_{1}(\mathrm{LO})$ stretch modes of short $B-\mathrm{O}$ bonds. The fwhm of the $\sim 95 \mathrm{meV}$ band would then be related to the spread of the $B-\mathrm{O}$ bond length distribution. The larger fwhm of the $\sim 95 \mathrm{meV}$ band in
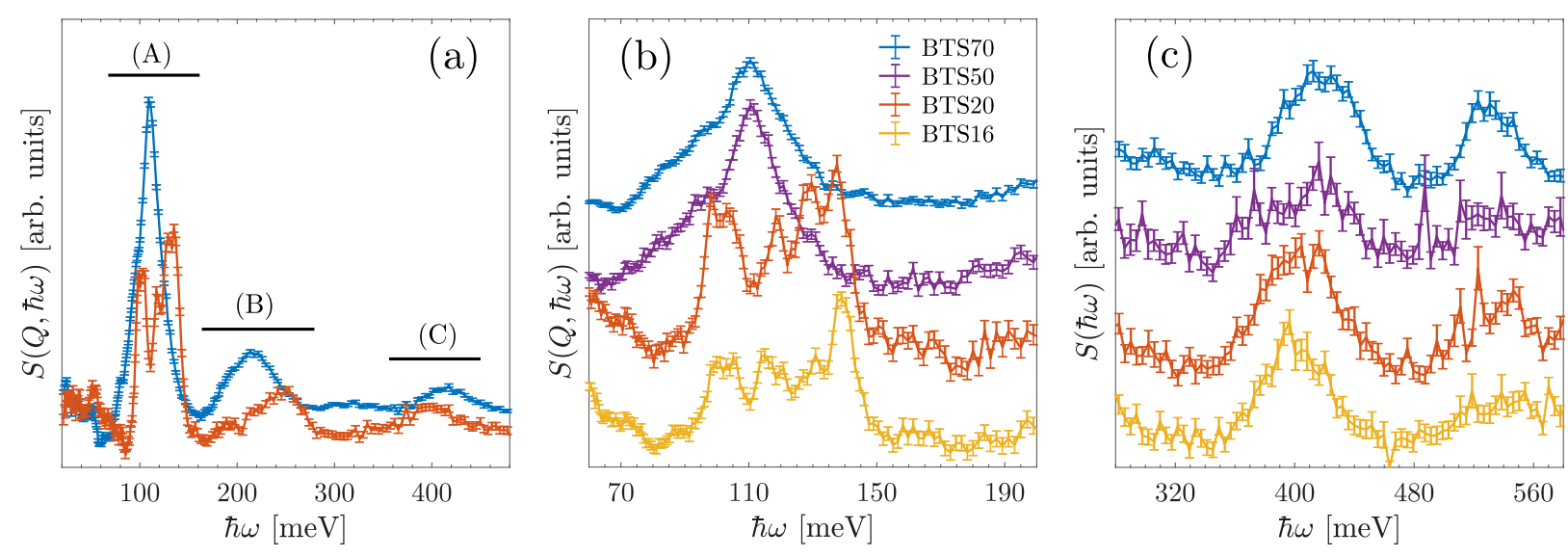

Figure 4. (a) IN1 Lagrange data. $S(Q \hbar \omega)$ of BTS70 (blue) and BTS20 (red). (b) TOSCA data. $S(Q \hbar \omega)$ of BTS70 (blue), BTS50 (purple), BTS20 (red), and BTS16 (yellow) over the $\delta(\mathrm{O}-\mathrm{H})$ mode region. Spectra are offset for clarity. (c) MAPS data. $S(\hbar \omega)$ of the four samples over the $\nu(\mathrm{O}-\mathrm{H})$ mode region (same color code as for part $\mathrm{b})$, obtained by integration of $S(Q \hbar \omega)$ over the interval $Q=[0-12] \AA^{-1}$. Spectra are vertically offset for clarity. 
BTS50 than in BTS70 is then consistent with the higher expected disorder in BTS50. The relatively lower intensity of the $\sim 95 \mathrm{meV}$ band in BTS16 and BTS20 than in the cubic phases may then be due to the reduced number of disordered $B$-sites. Furthermore, an additional band at $\sim 94 \mathrm{meV}$ is also observed in $\mathrm{BaZr}_{x} \mathrm{Ti}_{1-x} \mathrm{O}_{3}$ for Ti-rich compositions $(x \leq 0.6)$, and is interpreted as a signature of the relaxor phase. ${ }^{50,51}$ This may correspond in BTSX to the band observed at 100.8, 102.0, and $102.7 \mathrm{meV}$ for BTS50, BTS20, and BTS16, respectively.

Inelastic Neutron Scattering. The $S(Q \hbar \omega)$ of the BTS20 and BTS70 samples, measured on IN1 Lagrange, are shown in Figure 4a. The spectrum of the BTS70 sample shows strong similarities to other hydrated acceptor-doped cubic perovskite oxides. $^{37,39,40}$ It is characterized by three broad bands at 95-120 meV (A), 190-240 meV (B), and 380-440 $\mathrm{meV}$ (C), assigned, by comparison to said isotructural materials, ${ }^{37,39,40}$ to the fundamental $\delta(\mathrm{O}-\mathrm{H})$ modes, overtone of the $\delta(\mathrm{O}-\mathrm{H})$ modes, and fundamental $\nu(\mathrm{O}-\mathrm{H})$ modes, respectively. The broad nature of the bands is attributed in this family of compounds to inhomogeneous broadening caused by a distribution in the strength of the hydrogen bonds. By comparison, the spectrum of the hexagonal BTS20 sample shows several well-defined peaks over the $\delta(\mathrm{O}-\mathrm{H})$ region (Figure 4b), at $\hbar \omega=98,104,118,128$, and $137 \mathrm{meV}$, which suggest the presence of at least three distinct proton environments. Note the overall blue-shift of the $\delta(\mathrm{O}-\mathrm{H})$ band, and associated red-shift of the $\nu(\mathrm{O}-\mathrm{H})$ band, indicating in overall stronger hydrogen bonds in the hexagonal than in the cubic phase.

The $S(Q, \hbar \omega)$ over the $\delta(\mathrm{O}-\mathrm{H})$ mode region of the four samples, measured on TOSCA, are shown in Figure $4 \mathrm{~b}$. The two cubic samples, BTS50 and BTS70, have similar spectra, showing the broad band centered at about $\hbar \omega=110 \mathrm{meV}$, discussed previously. There is no argument, based on the structure or the spectra, which suggests that the nature of the proton sites may be different between these two materials. The spectra of the two hexagonal samples, BTS16 and BTS20, are also similar overall, showing distinct peaks. The major difference relates to the relative intensity of these peaks, associated with the relative occupation of the proton sites. The increase of the high-energy $\delta(\mathrm{O}-\mathrm{H})$ peak, at about $138 \mathrm{meV}$, indicates a higher population of strongly hydrogen-bonded protons in BTS16 than in BTS20.

The $S(\hbar \omega)$ over the extended $\nu(\mathrm{O}-\mathrm{H})$ modes region of the four samples, measured on MAPS, are shown in Figure 4c. The spectra are obtained by integration of the $S(Q \hbar \omega)$ data over the interval $Q=[0-12] \AA^{-1}$. The broad bands in the interval $350-450 \mathrm{meV}$ are tentatively assigned to the fundamental $\nu(\mathrm{O}-\mathrm{H})$ modes, and the bands at $\hbar \omega>500 \mathrm{meV}$ to combinations between fundamental wag and stretch modes (multiphonon contribution). The fundamental $\nu(\mathrm{O}-\mathrm{H})$ modes are found at about $420 \mathrm{meV}$ for BTS50 and BTS70, and 405 and $400 \mathrm{meV}$ for BTS20 and BTS16, respectively, which confirms the overall stronger hydrogen bonds in the hexagonal phases. It is also consistent with the greater amount of strongly hydrogen-bonded protons in BTS16 than in BTS20. Note the presence of a low energy shoulder on the $\nu(\mathrm{O}-\mathrm{H})$ band of BTS50 at about $380 \mathrm{meV}$, which may indicate that additional proton sites are present in BTS50 with respect to BTS70.

First-Principles Calculations. We have performed firstprinciples lattice dynamics calculations of pristine $\mathrm{BaTiO}_{3}$ with a cubic and hexagonal structure. The phonon eigenvectors at the $\Gamma$-point for the two structures are reported in Tables S1 and S2 in the Supporting Information, respectively.

The energies of the $\delta(\mathrm{O}-\mathrm{H})$ and $\nu(\mathrm{O}-\mathrm{H})$ bands are calculated in the relaxed structure of cubic $\mathrm{BaTiO}_{3}$ where a single proton, $\mathrm{OH}_{\mathrm{O}}^{\circ}$, is added to the vicinity of one of the (formerly equivalent) oxygen atoms. The hydroxide band energies are calculated at 71,116 , and $455 \mathrm{meV}$, which correspond, with respect to the $\mathrm{Ti}-\mathrm{O}-\mathrm{Ti}$ plane, to the in-plane wag motion $\left(\delta(\mathrm{O}-\mathrm{H})_{\|}\right)$, out-of-plane wag motion $\left(\delta(\mathrm{O}-\mathrm{H})_{\perp}\right)$, and stretch motion $(\nu(\mathrm{O}-\mathrm{H}))$, respectively. Considering the effect of $\mathrm{Sc}$, we calculated several structures containing both $\mathrm{OH}_{\mathrm{O}}^{\bullet}$ and $\mathrm{Sc}_{\mathrm{Ti}}^{\prime}$. The most stable structure is found with the scandium and the proton in close proximity. Note that the configuration with $\mathrm{Sc}$ and $\mathrm{OH}$ as second nearest neighbor is also rather likely, with an energy only $42 \mathrm{meV}$ higher. The hydroxide band energies are calculated at 71, 113, and 452 $\mathrm{meV}$, which suggests that the proton vibrational dynamics are largely unaffected by the presence of Sc.

For the hexagonal phases, several structures with distinct proton configurations are calculated (see Figure 5). The

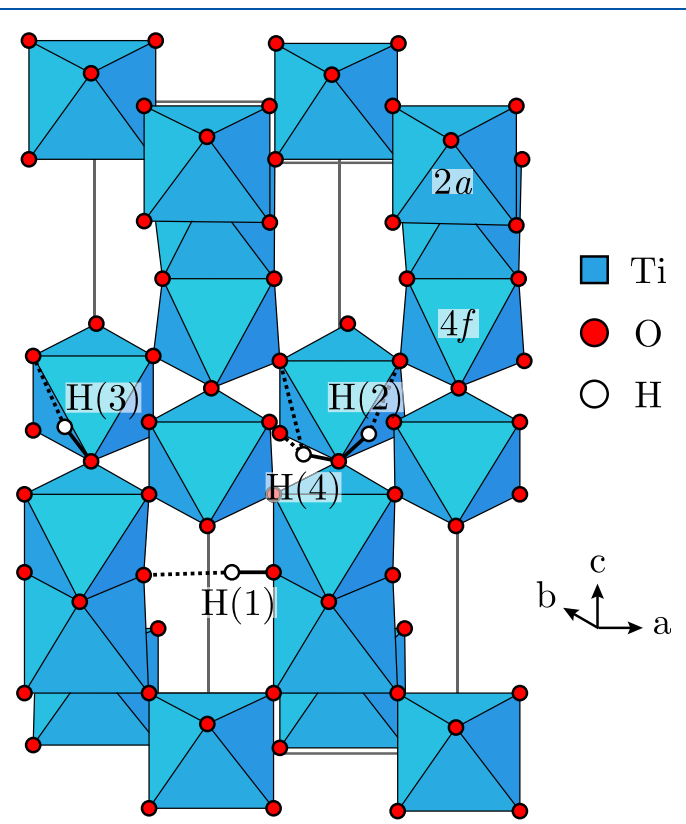

Figure 5. Scheme of the hexagonal $\mathrm{BaTiO}_{3}$ structure with the three most stable proton configurations, marked $\mathrm{H}(1), \mathrm{H}(2)$, and $\mathrm{H}(3)$. The more energetic $\mathrm{H}(4)$ site, discussed in the text, is also indicated. Barium atoms are omitted for clarity. Covalent bonds are shown as solid lines and hydrogen bonds as dashed lines.

geometry of the hydrogen bonds in the three most stable structures, their calculated vibrational energies, and the energy difference from the most stable structure, are reported in Table 2. The $\mathrm{H}(1)$ configuration is found the most stable, and is characterized by an interoctahedral hydrogen bond with a hydrogen bond angle close to $180^{\circ}$, in agreement with our previous work. ${ }^{27}$ Despite the relatively long hydrogen bond length, and because of its linear geometry, the calculated vibrational energies indicate that the hydrogen bond strength is superior to that of the cubic compound. The $\mathrm{H}(2)$ and $\mathrm{H}(3)$ configurations are associated with a hydrogen bond geometry comparable to the proton configuration in the cubic structure, on the edge of the $\mathrm{TiO}_{6}$ octahedra, yet also with stronger hydrogen bonds. Other structures, such as the one marked $\mathrm{H}(4)$ in Figure 5, involving hydrogen bond patterns on a face 
Table 2. Geometry of the Covalent Bonds (CB) and Hydrogen Bonds (HB) and Proton Vibrational Energies for the Three Most Stable Proton Configurations in the Calculation of Hexagonal $\mathrm{BaTiO}_{3}{ }^{a}$

$\begin{array}{llll} & \mathrm{H}(1) & \mathrm{H}(2) & \mathrm{H}(3) \\ \text { CB length }[\AA] & 0.98 & 1.03 & 1.02 \\ \text { HB length }[\AA] & 2.04 & 1.94 & 1.90 \\ \text { HB angle }[\mathrm{deg}] & 178 & 140 & 145 \\ \delta(\mathrm{O}-\mathrm{H})_{\|}[\mathrm{meV}] & 94 & 114 & 118 \\ \delta(\mathrm{O}-\mathrm{H})_{\perp}[\mathrm{meV}] & 134 & 130 & 125 \\ \nu(\mathrm{O}-\mathrm{H})[\mathrm{meV}] & 427 & 409 & 411 \\ \Delta E[\mathrm{meV}] & 0 & 317 & 355\end{array}$

${ }^{a}$ The difference in energy from the most stable configuration is also indicated.

of the $\mathrm{TiO}_{6}$ octahedra, with two acceptor oxygen atoms, are too energetic to be present in the real material. On the basis of energetic considerations, the experimental spectra should have a major contribution from the $\mathrm{H}(1)$ configuration and minor contributions from the $\mathrm{H}(2)$ and $\mathrm{H}(3)$ configurations.

Comparison between Experimental and Theoretical Results. The comparison between the Raman spectra of the cubic BTS70 and hexagonal BTS16 and the calculated energies for pristine $\mathrm{BaTiO}_{3}$ with cubic and hexagonal structures is shown in Figure 6. The region of the spectra corresponding to

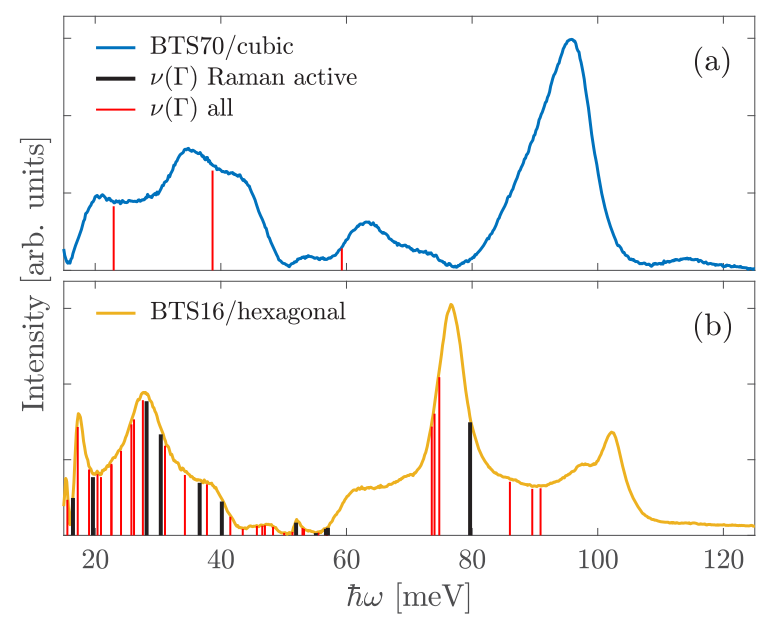

Figure 6. Comparison between the Raman spectra of cubic BTS70 (a) and hexagonal BTS16 (b) and calculated vibrational energies at the $\Gamma$-point, $\nu(\Gamma)$, marked by a thin red line. Raman active modes are marked by a thick black line. The intensity of the calculated modes is arbitrary.

the heavy elements, $\hbar \omega \leq 50 \mathrm{meV}$, is well matched by the calculations, with the main vibrational energies matching the peak positions. However, in the region of the spectra dominated by the oxygen modes, above $\hbar \omega \geq 50 \mathrm{meV}$, the agreement of the calculations with the spectra is poor, even when considering forbidden reflections made active by local disorder (red lines in Figure 6). This suggests that the inclusion of $\mathrm{OH}_{\mathrm{O}}^{\bullet}$ and $\mathrm{Sc}_{\mathrm{Ti}}^{\prime}$ into the structure drastically changes the dynamics of the oxygen atoms. Furthermore, the Raman active oxygen modes in the high energy regions (cf. Figure 3), which are induced by the Sc substitution, may be, to some extent, associated with the variation of hydrogen bond strength for protons in the crystal structure, as further discussed below.
Table 3 shows the vibrational energies observed in the INS spectra of cubic BTS70 and hexagonal BTS16 and the

Table 3. Measured Proton Vibrational Energies in Cubic BTS70 and Hexagonal BTS16, Together with the Calculated Proton Vibrational Energies in the Cubic and Hexagonal Defect Cell Models

\begin{tabular}{|c|c|c|c|c|}
\hline \multicolumn{5}{|c|}{ cubic } \\
\hline & & expt & \multicolumn{2}{|c|}{ calcd } \\
\hline$\delta(\mathrm{O}-\mathrm{H})_{\|}[\mathrm{meV}]$ & & - & \multicolumn{2}{|c|}{71} \\
\hline$\delta(\mathrm{O}-\mathrm{H})_{\perp}[\mathrm{meV}]$ & & 110 & \multicolumn{2}{|c|}{113} \\
\hline$\nu(\mathrm{O}-\mathrm{H})[\mathrm{meV}]$ & & 416 & \multicolumn{2}{|c|}{452} \\
\hline \multicolumn{5}{|c|}{ hexagonal } \\
\hline & expt & $\mathrm{H}(1)$ & $\mathrm{H}(2)$ & $\mathrm{H}(3)$ \\
\hline$\delta(\mathrm{O}-\mathrm{H})_{\|}[\mathrm{meV}]$ & 98 & 94 & - & - \\
\hline$\delta(\mathrm{O}-\mathrm{H})_{\|}[\mathrm{meV}]$ & 104 & - & 114 & - \\
\hline$\delta(\mathrm{O}-\mathrm{H})_{\|}[\mathrm{meV}]$ & 115 & - & - & 118 \\
\hline$\delta(\mathrm{O}-\mathrm{H})_{\perp}[\mathrm{meV}]$ & 121 & - & - & 125 \\
\hline$\delta(\mathrm{O}-\mathrm{H})_{\perp}[\mathrm{meV}]$ & 130 & - & 130 & - \\
\hline$\delta(\mathrm{O}-\mathrm{H})_{\perp}[\mathrm{meV}]$ & 139 & 134 & - & - \\
\hline$\nu(\mathrm{O}-\mathrm{H})[\mathrm{meV}]$ & 400 & 427 & 409 & 411 \\
\hline
\end{tabular}

calculated $\delta(\mathrm{O}-\mathrm{H})$ and $\nu(\mathrm{O}-\mathrm{H})$ bands for the cubic and hexagonal defect cells. Overall, we observe a good agreement between the experimental and calculated proton vibrational energies for the $\delta(\mathrm{O}-\mathrm{H})$ bands, while the $\nu(\mathrm{O}-\mathrm{H})$ energies are overestimated in the calculations, especially for the cubic structure. Note that the structural model considered here is $\mathrm{BaTiO}_{3}$, i.e. scandium-free (or a single-scandium atom for the cubic structure), which leads to pronounced disparities in terms of cell parameters between calculation and experiment, which could explain the differences in proton vibrational energies. Nonetheless, the tentative association of the experimental $\delta(\mathrm{O}-\mathrm{H})$ vibrational energies of BTS16 with the calculated ones, shown in Table 3, leads us to a description of the hexagonal BTSX where at least three distinct proton configurations may coexist: the $\mathrm{H}(1)$ configuration characteristic of the hexagonal structure, and the $\mathrm{H}(2)$ and $\mathrm{H}(3)$ configurations remnant of the cubic structure. Comparing the spectra of hexagonal BTS16 and BTS20, we observe that the band at $139 \mathrm{meV}$, assigned to the $\mathrm{H}(1) \delta(\mathrm{O}-\mathrm{H})_{\perp}$ mode, has increased intensity when the content of $\mathrm{Sc}$ is lower, i.e., when the material is further away from the phase transition, which supports our interpretation.

Regarding the effect of the scandium content across the entire substitution range, we observe with increasing $\mathrm{Sc}$ content an overall increase of the $\nu(\mathrm{O}-\mathrm{H})$ band energy, i.e., a decrease in hydrogen bond strength. This effect is likely related to the large difference in ionic radius between $\mathrm{Ti}^{4+}$ and $\mathrm{Sc}^{3+}, 60$ and $74 \mathrm{pm}$, respectively. As scandium substitutes preferentially in the $(2 a)$ sites (cf. Figure 5$),{ }^{27}$ and since we have calculated that $\mathrm{OH}_{\mathrm{O}}^{\bullet}$ and $\mathrm{Sc}_{\mathrm{Ti}}^{\prime}$ attract each other, we propose the following structural description. In the hexagonal phases, increasing the scandium content leads to an increase of the proportion of cubic-like proton environments, and a decrease of their hydrogen bond strength, until the phase transition to the cubic structure. In the cubic structure, remnants of hexagonal-type hydrogen bond configurations may still be present in $\mathrm{Ti}$ clusters (see the low-energy $\nu(\mathrm{O}-\mathrm{H}$ ) shoulder of BTS50 on Figure 4c), which disappear with increasing Sc content. As the $\mathrm{Ti}-\mathrm{OH}-\mathrm{Ti} / \mathrm{Sc}$ pattern becomes less likely, the hydrogen bond strength stabilizes at moderate- 
to-high Sc contents. Considering the superior bulk proton conducting properties of the cubic phases with respect to the hexagonal phases, by about 2 orders of magnitude, ${ }^{25}$ we infer that the presence of hexagonal-type proton-configurations, originating from Ti-rich domains, is unfavorable for the proton-conducting properties.

This result thus clearly highlights the benefits of highsymmetry structures with high-multiplicity sites to accommodate high proton conductivity in $\mathrm{BaTi}_{1-x} \mathrm{Sc}_{x} \mathrm{O}_{3} \mathrm{H}_{x}$ and, by extension, to other related proton-conducting oxides.

\section{CONCLUSIONS}

We have shown that the main factor impacting the local coordination environment and vibrational dynamics of protons in hydrated $\mathrm{BaTi}_{1-x} \mathrm{Sc}_{x} \mathrm{O}_{3} \mathrm{H}_{x}$ is the crystal structure, as protons occupy a single site in the cubic phase $(x=0.5$ and 0.7$)$ and three distinct sites in the hexagonal phase $(x=0.16$ and 0.2$)$. The main proton site in the hexagonal phase directly originates from the hexagonal arrangement and is characterized by interoctahedral hydrogen bonds, while the two additional sites are remnants of the cubic structure, with classic intraoctahedral geometries. The impact of the scandium content, besides on the absolute number of protons, appears limited to an overall decrease of the hydrogen bond strength with increasing Sc content, which is primarily attributed to the difference in ionic radius between $\mathrm{Sc}^{3+}$ and $\mathrm{Ti}^{4+}$. These results suggest that the significant drop of macroscopic proton conductivity at the cubic-to-hexagonal phase transition, rather than being due to the absolute number of protons, is predominantly due to the presence of stronger, more energetic hydrogen bonds in the hexagonal structure, which also involves proton sites with lower symmetry (lower multiplicity) than in the cubic structure. This insight showcases the favorable role of highsymmetry crystal structures and high-multiplicity proton sites to promote high proton conductivity in $\mathrm{Sc}$-doped $\mathrm{BaTiO}_{3}$ and, by extension, to other proton-conducting oxides.

\section{ASSOCIATED CONTENT}

\section{SI Supporting Information}

The Supporting Information is available free of charge at https://pubs.acs.org/doi/10.1021/acs.jpcc.0c01705.

Eigenvectors from the lattice dynamics calculations of cubic and hexagonal $\mathrm{BaTiO}_{3}(\mathrm{PDF})$

\section{AUTHOR INFORMATION}

\section{Corresponding Author}

Adrien Perrichon - Department of Chemistry and Chemical Engineering, Chalmers University of Technology, SE-412 96 Göteborg, Sweden; (1) orcid.org/0000-0003-0520-0469; Email: adrien.perrichon@physics.uu.se

\section{Authors}

Nico Torino - Department of Chemistry and Chemical Engineering, Chalmers University of Technology, SE-412 96 Göteborg, Sweden

Erik Jedvik Granhed - Department of Physics, Chalmers University of Technology, SE-41296 Göteborg, Sweden; (1) orcid.org/0000-0001-9119-9729

Yuan-Chih Lin - Department of Chemistry and Chemical Engineering, Chalmers University of Technology, SE-412 96 Göteborg, Sweden; 이이.org/0000-0002-0028-7481
Stewart F. Parker - ISIS Neutron and Muon Source, Science and Technology Facilities Council, Rutherford Appleton

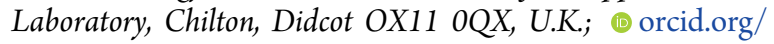
0000-0002-3228-2570

Mónica Jiménez-Ruiz - Institut Laue-Langevin, 38000 Grenoble, France; 이 orcid.org/0000-0002-9856-807X

Maths Karlsson - Department of Chemistry and Chemical Engineering, Chalmers University of Technology, SE-412 96 Göteborg, Sweden; 이이.org/0000-0002-2914-6332

Paul F. Henry - Department of Chemistry and Chemical Engineering, Chalmers University of Technology, SE-412 96 Göteborg, Sweden; ISIS Neutron and Muon Source, Science and Technology Facilities Council, Rutherford Appleton Laboratory,

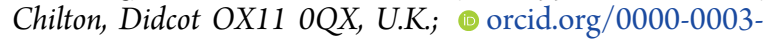
4714-6587

Complete contact information is available at:

https://pubs.acs.org/10.1021/acs.jpcc.0c01705

\section{Notes}

The authors declare no competing financial interest.

\section{ACKNOWLEDGMENTS}

This project was supported by the Swedish Research Council (Grant Nos. 2016-06968 and 2011-4887) and the Swedish Graduate School in Materials Science. The authors thank the Institut Laue-Langevin and the ISIS Neutron and Muon Source for access to neutron beam facilities. Access to the raw experimental neutron data are provided according to the ILL and ISIS Facility data policies; IN $1,{ }^{53}$ TOSCA, ${ }^{54}$ and MAPS. ${ }^{55}$ Simulations were performed on resources provided by the Swedish National Infrastructure for Computing (SNIC) at PDC Centre for High Performance Computing (PDC-HPC).

\section{REFERENCES}

(1) Malerød-Fjeld, H.; Clark, D.; Yuste-Tirados, I.; Zanón, R.; Catalán-Martinez, D.; Beeaff, D.; Morejudo, S. H.; Vestre, P. K.; Norby, T.; Haugsrud, R.; et al. Thermo-Electrochemical Production of Compressed Hydrogen From Methane With Near-Zero Energy Loss. Nature Energy 2017, 2, 923-931.

(2) Morejudo, S. H.; Zanón, R.; Escolástico, S.; Yuste-Tirados, I.; Malerød-Fjeld, H.; Vestre, P. K.; Coors, W. G.; Martínez, A.; Norby, T.; Serra, J. M.; et al. Direct Conversion of Methane to Aromatics in a Catalytic Co-Ionic Membrane Reactor. Science 2016, 353, 563-566.

(3) Iwahara, H.; Esaka, T.; Uchida, H.; Maeda, N. Proton Conduction in Sintered Oxides and its Application to Steam Electrolysis for Hydrogen Production. Solid State Ionics 1981, 3-4, 359-363.

(4) Sakai, T.; Matsushita, S.; Matsumoto, H.; Okada, S.; Hashimoto, S.; Ishihara, T. Intermediate Temperature Steam Electrolysis Using Strontium Zirconate-Based Protonic Conductors. Int. J. Hydrogen Energy 2009, 34, 56-63.

(5) Matsumoto, H.; Shimura, T.; Iwahara, H.; Higuchi, T.; Yashiro, K.; Kaimai, A.; Kawada, T.; Mizusaki, J. Hydrogen Separation Using Proton-Conducting Perovskites. J. Alloys Compd. 2006, 408-412, 456-462.

(6) Duan, C.; Tong, J.; Shang, M.; Nikodemski, S.; Sanders, M.; Ricote, S.; Almansoori, A.; O'Hayre, R. Readily Processed Protonic Ceramic Fuel Cells With High Performance at Low Temperatures. Science 2015, 349, 1321-1326.

(7) Kilner, J. A.; Burriel, M. Materials for Intermediate-Temperature Solid-Oxide Fuel Cells. Annu. Rev. Mater. Res. 2014, 44, 365-393.

(8) Norby, T.; Widerøe, M.; Glöckner, R.; Larring, Y. Hydrogen in Oxides. Dalton Trans. 2004, 19, 3012-3018.

(9) Kreuer, K. Proton-Conducting Oxides. Annu. Rev. Mater. Res. 2003, 33, 333-359. 
(10) Norby, T. Solid-State Protonic Conductors: Principles, Properties, Progress and Prospects. Solid State Ionics 1999, 125, 1-11. (11) Malavasi, L.; Fisher, C. A. J.; Islam, M. S. Oxide-Ion and Proton Conducting Electrolyte Materials for Clean Energy Applications: Structural and Mechanistic Features. Chem. Soc. Rev. 2010, 39, 43704387.

(12) Fabbri, E.; Pergolesi, D.; Traversa, E. Materials Challenges Toward Proton-Conducting Oxide Fuel Cells: A Critical Review. Chem. Soc. Rev. 2010, 39, 4355-4369.

(13) Kreuer, K.; Paddison, S. J.; Spohr, E.; Schuster, M. Transport in Proton Conductors for Fuel-Cell Applications: Simulations, Elementary Reactions, and Phenomenology. Chem. Rev. 2004, 104, 46374678.

(14) Kreuer, K. On the Development of Proton Conducting Materials for Technological Applications. Solid State Ionics 1997, 97, $1-15$.

(15) Iwahara, H.; Yajima, T.; Hibino, T.; Ozaki, K.; Suzuki, H. Protonic Conduction in Calcium, Strontium and Barium Zirconates. Solid State Ionics 1993, 61, 65-69.

(16) Katahira, K.; Kohchi, Y.; Shimura, T.; Iwahara, H. Protonic Conduction in $\mathrm{Zr}$-Substituted $\mathrm{BaCeO}_{3}$. Solid State Ionics 2000, 138, 91-98.

(17) Lü, J.; Wang, L.; Fan, L.; Li, Y.; Dai, L.; Guo, H. Chemical Stability of Doped $\mathrm{BaCeO}_{3}-\mathrm{BaZrO}_{3}$ Solid Solutions in Different Atmospheres. J. Rare Earths 2008, 26, 505-510.

(18) Quarez, E.; Noirault, S.; Caldes, M. T.; Joubert, O. Water Incorporation and Proton Conductivity in Titanium Substituted Barium Indate. J. Power Sources 2010, 195, 1136-1141.

(19) Jayaraman, V.; Magrez, A.; Caldes, M.; Joubert, O.; Ganne, M.; Piffard, Y.; Brohan, L. Characterization of Perovskite Systems Derived From $\mathrm{Ba}_{2} \mathrm{In}_{2} \mathrm{O}_{5} \square$ : Part I: The Oxygen-Deficient $\mathrm{Ba}_{2} \mathrm{In}_{2(1-x)} \mathrm{Ti}_{2 x} \mathrm{O}_{5+x} \square_{1-x}(0 \leq x \leq 1)$ Compounds. Solid State Ionics 2004, 170, 17-24.

(20) Jayaraman, V.; Magrez, A.; Caldes, M.; Joubert, O.; Taulelle, F.; Rodriguez-Carvajal, J.; Piffard, Y.; Brohan, L. Characterization of Perovskite Systems Derived From $\mathrm{Ba}_{2} \mathrm{In}_{2} \mathrm{O}_{5} \square$ : Part II: The Proton Compounds $\mathrm{Ba}_{2} \mathrm{In}_{2(1-x)} \mathrm{Ti}_{2 x} \mathrm{O}_{4+2 x}(\mathrm{OH})_{y}[0 \leq x \leq 1 ; y \leq 2(1-x)]$. Solid State Ionics 2004, 170, 25-32.

(21) Kreuer, K. D.; Adams, S.; Münch, W.; Fuchs, A.; Klock, U.; Maier, J. Proton Conducting Alkaline Earth Zirconates and Titanates for High Drain Electrochemical Applications. Solid State Ionics 2001, 145, 295-306.

(22) Rahman, S. M. H.; Knee, C. S.; Ahmed, I.; Eriksson, S. G.; Haugsrud, R. $50 \mathrm{~mol} \%$ Indium Substituted $\mathrm{BaTiO}_{3}$ : Characterization of Structure and Conductivity. Int. J. Hydrogen Energy 2012, 37, $7975-7982$

(23) Norberg, S. T.; Rahman, S. M. H.; Hull, S.; Knee, C. S.; Eriksson, S. G. The Proton Conducting Electrolyte $\mathrm{BaTi}_{0.5} \mathrm{In}_{0.5} \mathrm{O}_{2.75}$ : Determination of the Deuteron Site and its Local Environment. J. Phys.: Condens. Matter 2013, 25, 454214.

(24) Rahman, S. M. H.; Norberg, S. T.; Knee, C. S.; Biendicho, J. J.; Hull, S.; Eriksson, S. G. Proton Conductivity of Hexagonal and Cubic BaTi1-xScxO3- $\delta(0.1 \leq \mathrm{x} \leq 0.8)$. Dalton Trans. 2014, 43, 1505515064.

(25) Rahman, S. M. H.; Norberg, S. T.; Knee, C. S.; Biendicho, J. J.; Hull, S.; Eriksson, S. G. Proton Conductivity of Hexagonal and Cubic $\mathrm{BaTi}_{1-x} \mathrm{Sc}_{x} \mathrm{O}_{3-\delta}(0.1 \leq x \leq 0.8)$. Dalton Trans. 2014, 43, 1505515064.

(26) Rahman, S.; Ahmed, I.; Haugsrud, R.; Eriksson, S.; Knee, C. Characterisation of Structure and Conductivity of $\mathrm{BaTi}_{0.5} \mathrm{Sc}_{0.5} \mathrm{O}_{3-\delta}$. Solid State Ionics 2014, 255, 140-146.

(27) Torino, N.; Henry, P. F.; Knee, C. S.; Bjørheim, T. S.; Rahman, S. M. H.; Suard, E.; Giacobbe, C.; Eriksson, S. G. The Influence of Cation Ordering, Oxygen Vacancy Distribution and Proton Siting on Observed Properties in Ceramic Electrolytes: The Case of Scandium Substituted Barium Titanate. Dalton Trans. 2017, 46, 8387-8398.

(28) Torino, N.; Henry, P. F.; Knee, C. S.; Callear, S. K.; Smith, R. I.; Rahman, S. M. H.; Eriksson, S. G. Insight Into the Dehydration Behaviour of Scandium-Substituted Barium Titanate Perovskites via
Simultaneous In Situ Neutron Powder Thermodiffractometry and Thermogravimetric Analysis. Solid State Ionics 2018, 324, 233-240.

(29) Richard, D.; Ferrand, M.; Kearley, G. J. Analysis and Visualisation of Neutron-Scattering Data. J. Neutron Res. 1996, 4, $33-39$.

(30) Arnold, O.; Bilheux, J.; Borreguero, J.; Buts, A.; Campbell, S.; Chapon, L.; Doucet, M.; Draper, N.; Ferraz Leal, R.; Gigg, M.; et al. Mantid-Data Analysis and Visualization Package for Neutron Scattering and $\mu$ SR Experiments. Nucl. Instrum. Methods Phys. Res., Sect. A 2014, 764, 156-166.

(31) Blöchl, P. E. Projector Augmented-Wave Method. Phys. Rev. B: Condens. Matter Mater. Phys. 1994, 50, 17953-17979.

(32) Kresse, G.; Joubert, D. From Ultrasoft Pseudopotentials to the Projector Augmented-Wave Method. Phys. Rev. B: Condens. Matter Mater. Phys. 1999, 59, 1758-1775.

(33) Kresse, G.; Furthmüller, J. Efficiency of Ab-Initio Total Energy Calculations for Metals and Semiconductors Using a Plane-Wave Basis Set. Comput. Mater. Sci. 1996, 6, 15-50.

(34) Kresse, G.; Furthmüller, J. Efficient Iterative Schemes for $\mathrm{Ab}$ Initio Total-Energy Calculations Using a Plane-Wave Basis Set. Phys. Rev. B: Condens. Matter Mater. Phys. 1996, 54, 11169-11186.

(35) Perdew, J. P.; Burke, K.; Ernzerhof, M. Generalized Gradient Approximation Made Simple. Phys. Rev. Lett. 1996, 77, 3865-3868.

(36) Togo, A.; Tanaka, I. First Principles Phonon Calculations in Materials Science. Scr. Mater. 2015, 108, 1-5.

(37) Mazzei, L.; Perrichon, A.; Mancini, A.; Wahnström, G.; Malavasi, L.; Parker, S. F.; Börjesson, L.; Karlsson, M. Local Structure and Vibrational Dynamics in Indium-Doped Barium Zirconate. J. Mater. Chem. A 2019, 7, 7360-7372.

(38) Mazzei, L.; Perrichon, A.; Mancini, A.; Malavasi, L.; Parker, S. F.; Börjesson, L.; Karlsson, M. Local Coordination of Protons in Inand Sc-Doped $\mathrm{BaZrO}_{3}$. J. Phys. Chem. C 2019, 123, 26065-26072.

(39) Karlsson, M.; Ahmed, I.; Matic, A.; Eriksson, S. G. Short-Range Structure of Proton-Conducting $\mathrm{BaM}_{0.10} \mathrm{Zr}_{0.90} \mathrm{O}_{2.95}(\mathrm{M}=\mathrm{Y}$, In, Sc and Ga) Investigated With Vibrational Spectroscopy. Solid State Ionics 2010, 181, 126-129.

(40) Karlsson, M.; Björketun, M. E.; Sundell, P. G.; Matic, A.; Wahnström, G.; Engberg, D.; Börjesson, L.; Ahmed, I.; Eriksson, S.; Berastegui, P. Vibrational Properties of Protons in Hydrated BaIn $\mathrm{Zr}_{1-x} \mathrm{O}_{3-x / 2}$. Phys. Rev. B: Condens. Matter Mater. Phys. 2005, $72,94303$.

(41) Lock, A. J.; Bakker, H. J. Temperature Dependence of Vibrational Relaxation in Liquid $\mathrm{H}_{2} \mathrm{O}$. J. Chem. Phys. 2002, 117, $1708-1713$.

(42) Novak, A. Hydrogen Bonding in Solids Correlation of Spectroscopic and Crystallographic Data. Large Molecules 1974, 18, $177-216$.

(43) Miller, F. A.; Wilkins, C. H. Infrared Spectra and Characteristic Frequencies of Inorganic Ions. Anal. Chem. 1952, 24, 1253-1294.

(44) Venkateswaran, U. D.; Naik, V. M.; Naik, R. High-Pressure Raman Studies of Polycrystalline $\mathrm{BaTiO}_{3}$. Phys. Rev. B: Condens. Matter Mater. Phys. 1998, 58, 14256.

(45) Robins, L. H.; Kaiser, D. L.; Rotter, L. D.; Schenck, P. K.; Stauf, G. T.; Rytz, D. Investigation of the Structure of Barium Titanate Thin Films by Raman Spectroscopy. J. Appl. Phys. 1994, 76, 7487-7498.

(46) Begg, B. D.; Finnie, K. S.; Vance, E. R. Raman Study of the Relationship Between Room-Temperature Tetragonality and the Curie Point of Barium Titanate. J. Am. Ceram. Soc. 1996, 79, 26662672.

(47) Cao, S.-G.; Li, Y.; Wu, H.-H.; Wang, J.; Huang, B.; Zhang, T.-Y. Stress-Induced Cubic-to-Hexagonal Phase Transformation in Perovskite Nanothin Films. Nano Lett. 2017, 17, 5148-5155.

(48) Nyutu, E. K.; Chen, C.-H.; Dutta, P. K.; Suib, S. L. Effect of Microwave Frequency on Hydrothermal Synthesis of Nanocrystalline Tetragonal Barium Titanate. J. Phys. Chem. C 2008, 112, 9659-9667.

(49) Kirianov, A.; Ozaki, N.; Ohsato, H.; Kohzu, N.; Kishi, H. Studies on the Solid Solution of $\mathrm{Mn}$ in $\mathrm{BaTiO}_{3}$. Jpn. J. Appl. Phys. 2001, 40, 5619. 
(50) Karan, N. K.; Katiyar, R. S.; Maiti, T.; Guo, R.; Bhalla, A. S. Raman Spectral Study of $\mathrm{Zr}^{4+}$-Rich $\mathrm{BaZr}_{x} \mathrm{Ti}_{1-x} \mathrm{O}_{3}(0.50 \leq x \leq 1.00)$ Phase Diagram. J. Raman Spectrosc. 2009, 40, 370-375.

(51) Farhi, R.; El Marssi, M.; Simon, A.; Ravez, J. A Raman and Dielectric Study of Ferroelectric Ceramics. Eur. Phys. J. B 1999, 9, 599-604.

(52) Sidorov, T. A. Structure of $\mathrm{BaTiO}_{3}$ Phases is Studied by Comparing Neutron Diffraction and Raman Spectroscopy Data. Russ. J. Inorg. Chem. 2011, 56, 1957-1966.

(53) Perrichon, A.; Jiménez-Ruiz, M.; Torino, N.; Rahman, S. M. H.; Karlsson, M. Experimental Data From IN1 Lagrange 2018, DOI: 10.5291/ILL-DATA.7-03-164.

(54) Perrichon, A.; Parker, S. F.; Torino, N.; Rahman, S. M. H.; Karlsson, M. Experimental Data From TOSCA. STFC ISIS Neutron and Muon Source 2018, DOI: 10.5286/ISIS.E.RB1810624.

(55) Perrichon, A.; Parker, S. F.; Torino, N.; Rahman, S. M. H.; Karlsson, M. Experimental Data From MAPS. STFC ISIS Neutron and Muon Source 2018, DOI: 10.5286/ISIS.E.RB1810628. 